\title{
Identifying appropriate protected areas for endangered fern species under climate change
}

\author{
Chun-Jing Wang, Ji-Zhong Wan, Zhi-Xiang Zhang and Gang-Min Zhang*
}

*Correspondence:
gary1967@bjfu.edu.cn
School of Nature
Conservation, Beijing Forestry
University, Beijing 100083,
China

*Correspondence: gary1967@bjfu.edu.cn Conservation, Beijing Forestry China

\begin{abstract}
The management of protected areas (PAs) is widely used in the conservation of endangered plant species under climate change. However, studies that have identified appropriate PAs for endangered fern species are rare. To address this gap, we must develop a workflow to plan appropriate PAs for endangered fern species that will be further impacted by climate change. Here, we used endangered fern species in China as a case study, and we applied conservation planning software coupled with endangered fern species distribution data and distribution modeling to plan conservation areas with high priority protection needs under climate change. We identified appropriate PAs for endangered fern species under climate change based on the IUCN protected area categories (from la to $\mathrm{VI}$ ) and planned additional PAs for endangered fern species. The high priority regions for protecting the endangered fern species were distributed throughout southern China. With decreasing temperature seasonality, the priority ranking of all endangered fern species is projected to increase in existing PAs. Accordingly, we need to establish conservation areas with low climate vulnerability in existing PAs and expand the conservation areas for endangered fern species in the high priority conservation regions.
\end{abstract}

Keywords: Conservation area, Endangered fern species, Climate change, Species distribution modeling, Conservation planning software, China

\section{Background}

Climate change has had a profound effect on biodiversity and can result in the migration, adaption, and extinction of species, as well as make it harder to protect endangered species (Pearson and Dawson 2003; Hampe and Petit 2005; Dawson et al. 2011; Chen 2013). Some studies have shown that changes in population and species structure may alter distributions of species diversity, affect habitats and thus induce responses in the phenotypic plasticity of individuals and populations, and change the distribution or fragmentation of habitats (Jackson and Sax 2010; Sgro et al. 2011; Zhang et al. 2014a; Chung et al. 2015). This ultimately reduces species diversity and results in a loss of biodiversity (Sgro et al. 2011; Bradford and Warren 2014).

Ferns are vascular plants that reproduce and disperse via spores (Graf 1999). A number of endangered fern species (EFS) have been seriously impacted by climate change and are in danger of extinction. However, current protection measures do not adequately 
support the conservation of EFS (http://www.iucnredlist.org/). Therefore, EFS conservation efforts are urgently required under climate change.

These protection issues can be addressed by the establishment of additional protected areas (PAs; Chape et al. 2005; Chen 2007). The future effectiveness of PAs is limited because climate change could drive endangered plant species out of PAs, resulting in a loss of their conservation function for endangered species (Araújo et al. 2011; Yu et al. 2014; Wang et al. 2016). A number of studies have suggested the integration of climate change into conservation planning for endangered plant species (Hannah et al. 2002; Araújo et al. 2011; Dawson et al. 2011). However, studies focusing on EFS conservation in conjunction with climate change are rare. Target seven of the Global Strategy for Plant Conservation (GSPC) has shown at least $75 \%$ of known threatened plant species would be conserved in situ from 2011 to 2020 (https://www.cbd.int/gspc/). However, conservationists and government managers cannot effectively establish more PAs for EFS because of limited funds and manpower (Leader-Williams and Albon 1988; Zhang et al. 2014a). Hence, it is necessary for conservationists to expand PAs to accommodate EFS, assess the ability of existing protected areas to conserve EFS, and determine the climatic features of these PAs. Thus, we aimed to determine the areas and number of wild EFS populations through field investigations and to plan to appropriately expand protected areas for EFS.

Conservation planning software and species distribution modeling (SDM) has been widely used in biological conservation, ecological restoration, and the planning of PAs (Summers et al. 2012; Chen 2013; Meller et al. 2014). Researchers have predicted the potential geographical distributions of endangered species, determined priority conservation areas for these species, and established a model-based evaluation system for the conservation of biodiversity in PAs by using conservation planning software and SDM (Di Minin and Moilanen 2012; Summers et al. 2012; Di Minin and Moilanen 2014; Wan et al. 2016). Conservation planning software coupled with SDM could be used to identify appropriate protected areas for EFS and determine priority conservation areas under climate change that are not covered by existing PAs (Di Minin et al. 2013).

We used the EFS of China as a case study because (1) EFS are widely distributed across a range of latitudes, (2) China contains a rich diversity of EFS, and (3) EFS conservation management is urgent because there are few PAs supporting EFS in China. The main objective of our study was to identify appropriate conservation areas for EFS under climate change based on conservation priority rankings computed with conservation planning software. To achieve this objective, we performed two tasks: (1) an evaluation of the ability of PAs to conserve EFS under climate change using Zonation (a common conservation planning software tool) and (2) a determination of the climatic features of PAs with high priority rankings. First, we used SDM in Maxent to model the potential distribution of EFS in China under climate change. Second, we used Zonation to plan priority conservation areas for EFS based on this potential distribution. Third, geographical information system (GIS) was used to compute the ability of protected areas to conserve EFS under climate change and explore the relationship between climate change and priority conservation areas in PAs. 


\section{Methods}

\section{PAs in China}

Data from the World Database on Protected Areas (WDPA) were used to identify the PAs in China with areas greater than $4.3 \mathrm{~km}$ at the equator that were suitable for analysis in this study (http://www.protectedplanet.net/). We classified 642 Chinese PAs into five groups based on the IUCN protected area categories: Category Ia, strict nature reserve; Category II, national park; Category IV, habitat/species management area; Category V, protected landscape/seascape; and Category VI, protected area with sustainable use of natural resources. Based on WDPA database, there are no PAs belonging to Category Ib (wilderness area) and Category III (natural monument or feature; http://www.protectedplanet.net/).

\section{Species data}

EFS were selected from the List of National Key Protected Wild Plants approved by the State Council of China (http://www.gov.cn/gongbao/content/2000/content_60072. $\mathrm{htm})$. Occurrence localities that contain EFS were identified from the following three sources: (1) The Global Biodiversity Information Facility (GBIF; http://www.gbif.org/); (2) the Chinese Virtual Herbarium (CVH; http://www.cvh.org.cn/); and (3) 175 scientific research reports detailing national nature reserves (more detailed information is provided in the "Acknowledgements" section). Although some studies did not report the geographical coordinates of some species, we were able to translate the recorded locations of species into latitudes and longitudes using Google Earth and ArcGIS 10.2 (Esri; Redlands, CA, USA) based on (1) the detailed location and habitat descriptions of species from the research reports; (2) vegetation information about species from the 1:1 Million Vegetation Atlas of China (Hou 2001); and (3) the locations of species within 10 -arc-minute grid cells (equivalent to $16 \mathrm{~km}$ at the equator) to avoid any georeferencing errors (Zhang et al. 2014b). These three factors limited the extent of species occurrences to roughly 10-arc-minute grids. Finally, we selected 16 EFS with more than five occurrence localities as the input dataset for SDM (Pearson et al. 2007). We could not identify wild populations of some species owing to limited occurrence localities. For practical purposes, we focused on potential distributions of EFS with known wild populations.

\section{Modeling potential distributions of species}

Four contemporary bioclimatic variables at a 10-arc-minute spatial resolution $(16 \mathrm{~km}$ at the equator) were used to model potential distributions of species, and these climatic data were obtained from the WorldClim database (Additional file 1: Table S1; http:// www.worldclim.org/). The resulting four bioclimatic variables were related to the distribution and physiological performance of plants. Four projected bioclimatic variables, corresponding to the present-day variables, were assessed using the mean grid maps of three global climate models (GCMs), including mohc_hadgem2, csiro_mk3_6_0, and cccma_canesm 2 analogue data (corresponding to 2070-2099, or roughly the $2080 \mathrm{~s}$ ), and obtained from the International Centre for Tropical Agriculture (http://ccafs-climate. org). Representative concentration pathways (RCPs) 4.5 (mean, 780 ppm; range, 5951005 by 2100; low concentration scenario) and 8.5 (mean, 1685 ppm; range, 1415-1910 by 2100; high concentration scenario) were used to model future potential distributions 
of species. RCP 8.5 projections differ from RCP 4.5 projections because of higher cumulative concentrations of carbon dioxide and other largely anthropogenic greenhouse gas pollutants that alter the pattern of climate change (http://www.ipcc.ch/report/ar5/).

We used Maxent to model the projected EFS distributions in China under climate change (Merow et al. 2013). All grids were regarded as a possible distribution space according to maximum entropy (Elith et al. 2011; Merow et al. 2013). For the map grids predicted using Maxent, cell values of one represented the highest possibility of containing the species, while values close to zero represented the lowest possibility. Furthermore, projected EFS distribution areas were effectively determined using the contemporary climate conditions of the present-day sites that contain these individual EFS (Warren and Seifert 2011).

Maxent settings used in this analysis included the following: (1) the regularization multiplier (beta) was set to 1.5 for producing a smooth and general response that could be modeled in a biologically realistic manner (Shcheglovitova and Anderson 2013); (2) the maximum number of background points was 10,000 (Merow et al. 2013); (3) a fourfold cross-validation approach was used for removing bias with respect to recorded occurrence points, namely, $75 \%$ of occurrence points were used for training and $25 \%$ for the actual test (Li and Guo 2013); (4) a jackknife test was used in Maxent to analyze the importance of different climatic factors (Merow et al. 2013); and (5) all other settings used were the same as those described by Elith et al. (2011) and Merow et al. (2013). We projected the importance of climatic variables to potential distributions of species based on the results of the jackknife test (Merow et al. 2013).

Receiver operating characteristic (ROC) curves summarize each value of the prediction result as a possible analysis threshold. The precision of the model was evaluated by calculating the area under the ROC Curve (AUC). The models were either graded as poor (AUC $<0.8$ ), fair $(0.8<$ AUC $<0.9)$, good $(0.9<$ AUC $<0.95)$, or very good $(0.95<$ AUC < 1.0; Adhikari et al. 2012). Using the methods described by Calabrese et al. (2014), we computed predicted species richness under current, low, and high concentration scenarios by superimposing the weighted potential distribution of species for each grid. Then we selected some PAs that currently contain EFS (based on scientific surveys conducted in national nature reserves) and used linear-regression analyses to analyze the relationship between mean predicted species richness of grids and observed species richness in PAs in order to evaluate model precision at the PA scale. A significant relationship between these values is an important precondition for computing the priority ranking of the EFS. Here, we could not use all of the PAs from WDPA because of a lack of data. Hence, we only included the PAs for which species occurrence data was recorded in all data sources (i.e., GBIF, CVH, and scientific research reports by national nature reserves).

\section{Evaluating the ability of PAs to conserve EFS}

First, we used Zonation software to identify priority conservation areas for EFS in China. Zonation is a publicly available framework and software for grid-based and large-scale spatial conservation prioritization (Meller et al. 2014). It has been used for evaluating conservation areas and conservation planning under climate change scenarios (Summers et al. 2012). Using Zonation, we obtained the priority ranking of each grid for EFS. 
We focused on the connectivity between the current and future potential EFS distributions (under the low and high concentration scenarios) and considered the influence of climate change on future species richness when selecting potential sites for nature reserves (Lehtomäki and Moilanen 2013; Wan et al. 2015). Using the original core-area cell removal rule, we established spatial priorities and calculated the marginal loss of each grid, which we then used to determine if a conservation goal had been met (i.e., that a given proportion of distributions for all of the species with the high priority ranking would be protected; Lehtomäki and Moilanen 2013; Wang et al. 2015). Current and future species richness of EFS were weighted equally in our analysis (including under low and high concentration scenarios), and we used a warp factor of 100 (Wan et al. 2014, 2015; Wang et al. 2015). For the grid maps of priority conservation areas under low and high concentration scenarios, 10.0-arc-minutes resolution data were aggregated at 2.5-arc-minutes resolution using ArcGIS 10.2 (Esri; Redlands, CA, USA) to identify priority conservation areas in PAs. We also used ArcGIS 10.2 (Esri; Redlands, CA, USA) to extract and compute the priority ranking of all EFS and the average values of the most important climatic variables for PAs.

We computed the overall priority ranking of all EFS for each PA using the following equation:

$$
S_{t}=\sum_{j=1}^{n} P_{j} / A
$$

where $S_{t}$ represents the overall priority ranking of all EFS in PA $t, P_{j}$ represents the priority ranking value of all EFS in grid $j$ based on PA $t$, and $A$ represents the number of grids in PA $t$.

Finally, we used linear-regression analyses to compute the relationship between the average values of the most important climatic variables and priority ranking of all EFS for PAs. We also computed the mean priority ranking of all EFS for each group of PAs based on the IUCN protected area.

\section{Results}

We predicted the potential distributions of 16 EFS in China under current, low, and high concentration scenarios (Additional file 2: Fig. S1). Based on AUC values, our prediction model performed well for all species: the AUC value of each species was over 0.9 (mean value, 0.974; Table 1). We also found that there was a significant relationship between the mean predicted species richness of grids and the observed species richness in PAs $(R=0.470 ; P<0.05$; Fig. 1). Based on the jackknife test in Maxent, we found that the most important climatic variable was temperature seasonality (average contribution to EFS distributions, 59.8; Table 1). Furthermore, there was a significant relationship between priority ranking of all EFS and temperature seasonality $\left(R^{2}=0.7002 ; P<0.001\right.$; Fig. 2) indicating that with decreasing temperature seasonality the priority ranking of all the EFS would increase in PAs (Fig. 2).

We found that the regions with high priority ranking for all EFS were distributed throughout southern China (Fig. 3). This is consistent with the present-day distribution of occurrence localities of species we collected. Tawushan (Sichuan), Taitung Hungyeh 
Table 1 Endangered fern species, AUC values, and jackknife test results

\begin{tabular}{llrrrrr}
\hline Names & Family & AUC & Bio1 & Bio4 & Bio12 & Bio15 \\
\hline Cibotium barometz & Dicksoniaceae & 0.978 & 4.0 & 49.8 & 25.3 & 20.8 \\
Archangiopteris henryi & Angiopteridaceae & 0.996 & 99.1 & 0.9 & 0.0 & 0.0 \\
Sorolepidium glaciale & Dryopteridaceae & 0.976 & 0.6 & 58.3 & 0.0 & 41.2 \\
Helminthostachys zeylanica & Helminthostachyaceae & 0.989 & 1.0 & 73.6 & 0.1 & 25.3 \\
Isoetes sinensis & Isoetaceae & 0.965 & 0.2 & 2.5 & 9.8 & 87.5 \\
Ceratopteris thalictroides & Parkeriaceae & 0.947 & 16.7 & 46.3 & 24.2 & 12.8 \\
Neocheiropteris palmatopedata & Polypodiaceae & 0.992 & 0.0 & 93.4 & 6.6 & 0.0 \\
Alsophila costularis & Cyatheaceae & 0.992 & 0.0 & 92.5 & 0.1 & 7.4 \\
Alsophila denticulata & Cyatheaceae & 0.967 & 2.3 & 43 & 29 & 25.7 \\
Alsophila gigantea & Cyatheaceae & 0.984 & 1.6 & 63.2 & 7.2 & 28 \\
Alsophila loheri & Cyatheaceae & 0.999 & 0.0 & 94.7 & 5.3 & 0.0 \\
Alsophila metteniana & Cyatheaceae & 0.967 & 3.0 & 51.9 & 29.9 & 15.2 \\
Alsophila podophylla & Cyatheaceae & 0.959 & 1.5 & 65.9 & 26.2 & 6.3 \\
Alsophila spinulosa & Cyatheaceae & 0.967 & 1.4 & 66.8 & 28.3 & 3.5 \\
Sphaeropteris lepifera & Cyatheaceae & 0.964 & 0.4 & 88.8 & 10.4 & 0.4 \\
Brainea insignis & Blechnaceae & 0.942 & 1.9 & 65.9 & 25.8 & 6.5 \\
Mean & & 0.974 & 8.4 & 59.8 & 14.3 & 17.5 \\
\hline
\end{tabular}

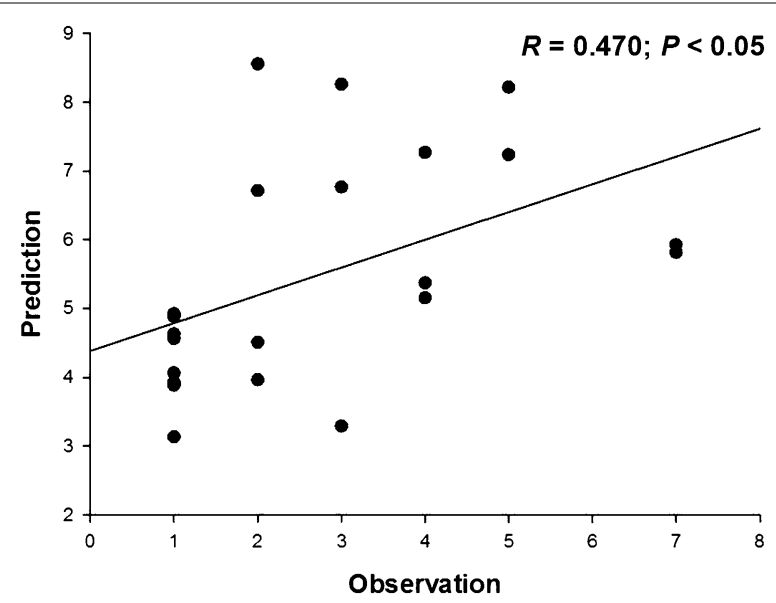

Fig. 1 The relationship between mean predicted species richness of grids and observed species richness at the scale of protected areas. Prediction mean predicted species richness of grids; Observation observed species richness

Village Taiwan Cycas (Taiwan), Yushan (Taiwan), Chuyunshan (Taiwan), and Nanlin (Hainan) PAs had high priority rankings for all EFS (Fig. 4a). These PAs are projected to effectively converse EFS. Furthermore, these PAs exhibit low temperature seasonality (Fig. 4b). We found that national parks and protected areas that permit the sustainable use of natural resources have a higher priority ranking for all EFS than strict nature reserves, habitat/species management areas, and protected landscapes/seascapes (Fig. 5). The PAs of habitat/species management areas have the lowest priority rankings for all EFS (Fig. 5). 


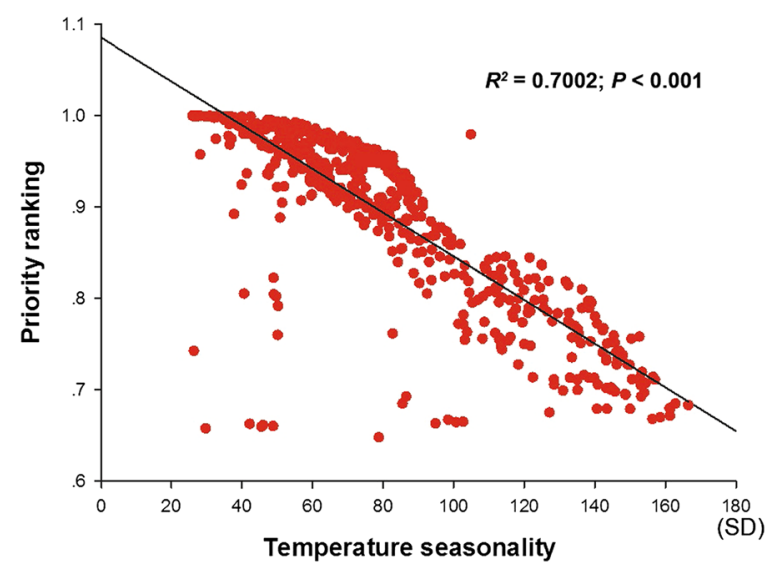

Fig. 2 The relationship between priority rankings of endangered fern species and temperature seasonality. Priority ranking the priority ranking of a single endangered fern species

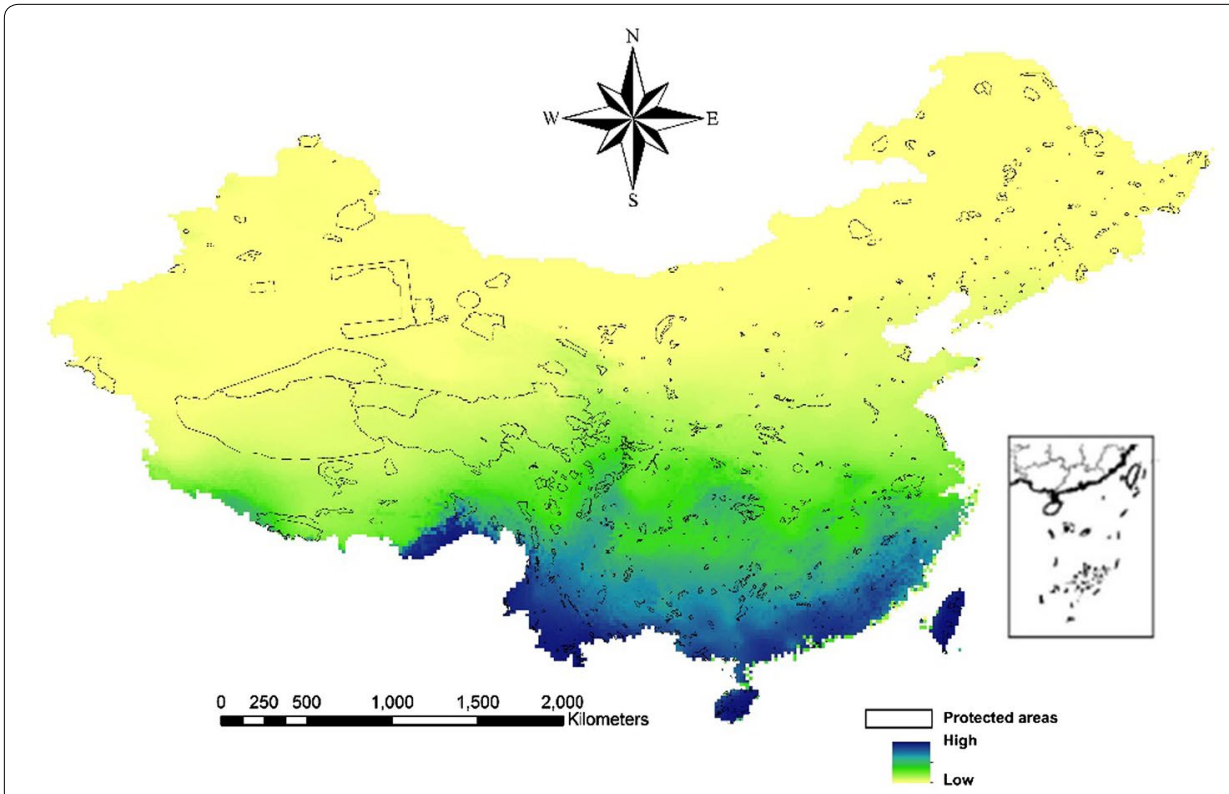

Fig. 3 A priority ranking map for endangered fern species in China. The color distribution from light to dark represents increasing priority ranking

\section{Discussion}

This study establishes a large-scale evaluation system for EFS based on the predicted impact of climate change. We suggest the use of robust results of potential species distributions to identify appropriate protected areas for EFS under different climate change models. To achieve this objective, we used two test methods for the potential EFS distributions. First, AUC provides important references that were used to assess the performance of Maxent, and then we tested the robustness of predicted species richness modeled by Maxent at the PA scale based on the relationship between predicted species richness and observed species richness of PAs (Pouteau et al. 2015). Previous studies have shown that the relationship between the predicted species richness of grids and the 


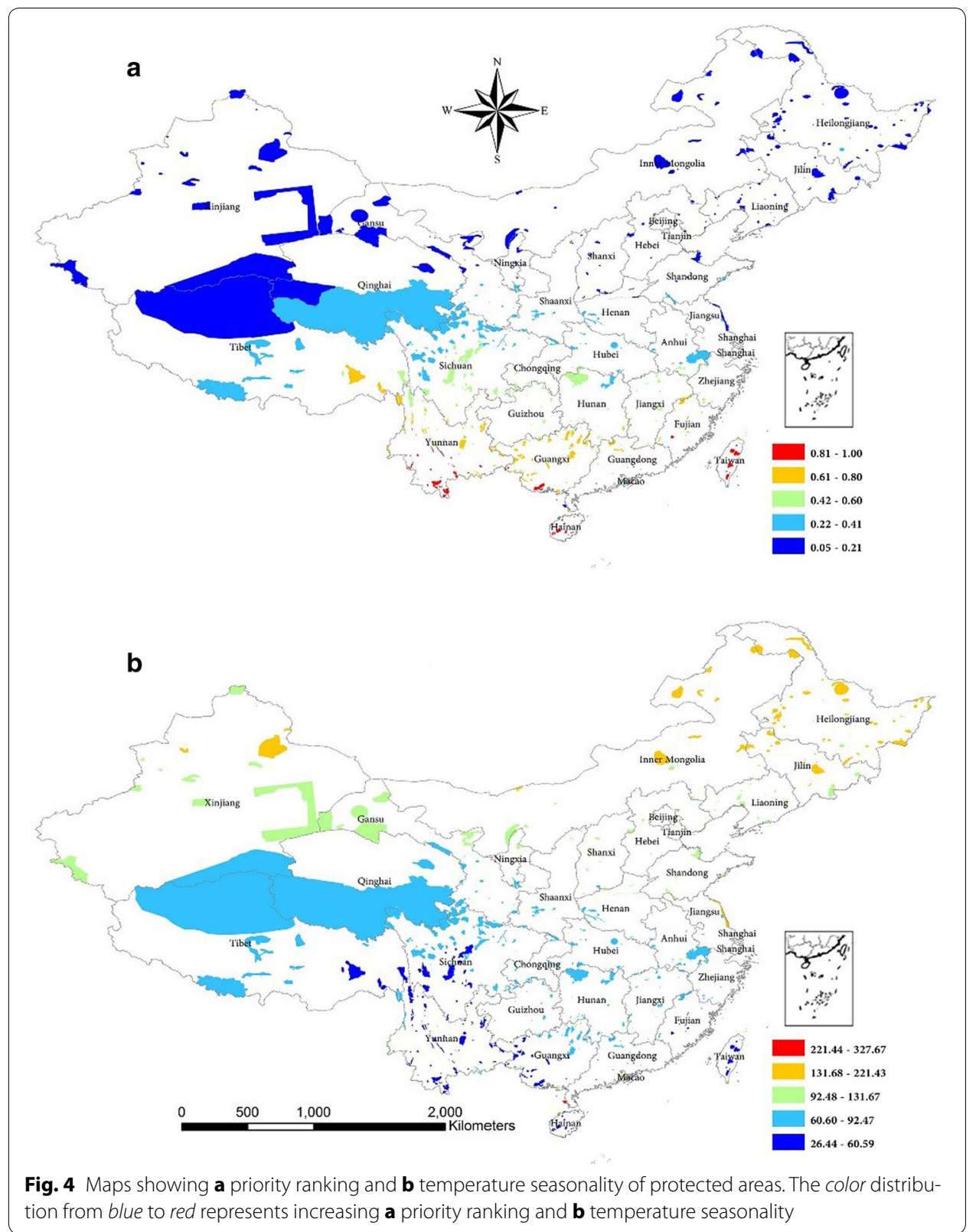

observed species richness is robust at the grid scale (Royle et al. 2012; Cao et al. 2013; Pouteau et al. 2015). However, the limits of occurrence locality data could not perfectly support this test method at the grid scale (Chen 2013). Hence, in addition to our main objectives, we also validated that the predicted species richness based on projected species distributions was robust at the PA scale.

Conservationists and government managers have begun to integrate climate change into conservation management (Lawler 2009; Heller and Zavaleta 2009; Dawson et al. 2011). This is likely a consequence of the increased understanding that climate change could drive potential distributions of plant species out of existing PAs, such that these PAs could lose their function of conserving endangered species (Araújo et al. 2011). 


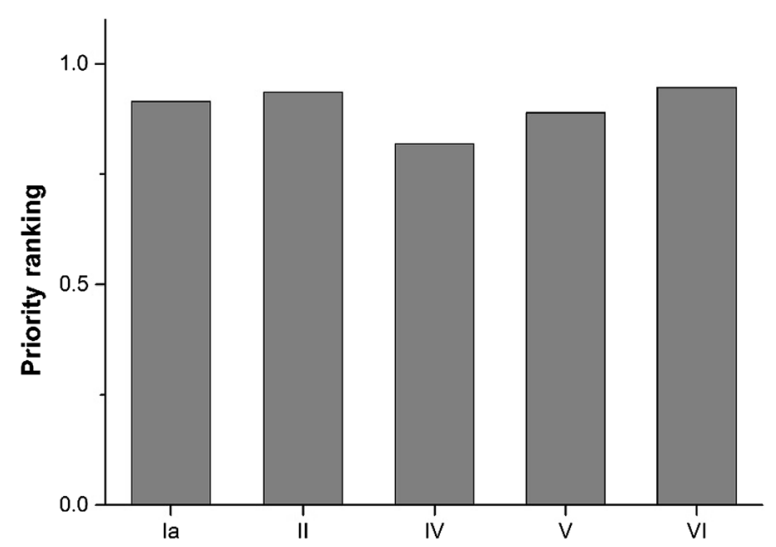

Fig. 5 The priority ranking of protected areas based on IUCN protected area categories. Priority ranking the mean priority ranking of endangered fern species in China

Previous studies have shown that the assessment of the ability of existing PAs to conserve endangered plant species can be an effective reference for enhancing the conservation of endangered plant species (Araújo et al. 2011; Wan et al. 2014; Yu et al. 2014; Wang et al. 2016). Accordingly, action is required to establish conservation areas of low climate vulnerability for EFS in existing PAs (Gillson et al. 2013). However, the conservation functions of many existing Chinese PAs are primarily intended to protect forest ecosystems and endangered animals (http://datacenter.mep.gov.cn/). There is a substantial opportunity to utilize these existing PAs to conserve EFS. We should therefore focus on national parks and protected areas that exhibit a sustainable use of natural resources in China. National parks are similar to wilderness areas in size and in their ecosystem protection function. Protected areas that sustainably use natural resources are focused on establishing mutually beneficial arrangements for nature conservation and the sustainable management of natural resources (http://www.iucn.org/). However, these two types of PAs are affected by human disturbance. Hence, in situ conservation areas should be separated from areas with a high density of human activities (Ravenel and Redford 2005; Wang et al. 2015). The PA priority rankings indicate we have selected appropriate PAs-i.e., Tawushan (Sichuan), Taitung Hungyeh Village Taiwan Cycas (Taiwan), Yushan (Taiwan), Chuyunshan (Taiwan) and Nanlin (Hainan)—for conducting detailed EFS investigations and enhancing in situ EFS conservation. In order to continue supporting EFS, these PAs need to have low temperature seasonality. Hence, it is necessary to integrate projected changes in temperature seasonality into the future EFS conservation efforts.

Some studies have suggested the expansion of PAs to allow for distribution changes under climate change (Heller and Zavaleta 2009; Lawler 2009; Araújo et al. 2011; Dawson et al. 2011). However, limited manpower and financial resources constrain the development of additional PAs (Leader-Williams and Albon 1988; Wang et al. 2016). Hence, we need to identify appropriate protected areas for EFS under climate change and develop more protected areas for EFS efficiently (Chen 2007; Heller and Zavaleta 2009). Our results shown in Figs. 3 and 4 indicate the need to establish a network of PAs that facilitate the exchange of EFS among PAs under climate change; conservation planning 
software could address this need (Di Minin and Moilanen 2012; Summers et al. 2012; Di Minin and Moilanen 2014). Sharafi et al. (2012) used Zonation to identify areas outside of existing PAs that efficiently cover gaps in biodiversity features and appropriately expand conservation areas in Victoria, Australia. This study provides a useful model for conservation efforts in China. Our suggestion is to strongly consider connectivity among existing PAs and to establish conservation areas for EFS in the regions among PAs with high priority rankings.

\section{Conclusion}

Our findings show that with decreasing temperature seasonality, national parks and protected areas in which natural resources are sustainably used had the highest priority ranking for all of the analyzed EFS. To reduce the negative impact of climate change on EFS, we should take immediate actions, such as establishing conservation areas with low climate vulnerability for EFS in existing PAs and expanding conservation areas for EFS into the regions with high priority rankings. We hope to use a large-scale priority ranking evaluation for the areas that are suitable for EFS conservation in order to promote the development of global conservation planning for threatened plant species. However, our study had some limitations, as we required more detailed data on climate and species distributions than was sometimes available. In future research, we will construct more accurate maps of appropriate conservation areas for EFS under climate change with appropriately richer data. Immediate EFS conservation actions should be considered in future worldwide studies.

\section{Additional files}

Additional file 1: Table S1. Environmental variables. Environmental variables were used as environmental layers to characterize the current distribution and predict the potential distribution of endangered fern species using Maxent; $\mathrm{C}$ of $\mathrm{V}$ represents the coefficient of variation; SD represents Standard Deviation.

Additional file 1: Fig. S1. The contemporary and projected distributions of endangered fern species in the (a) current, (b) low, and (c) high concentration scenarios.

Authors' contributions

C-JW, J-ZW, Z-XZ and G-MZ contributed to modelling, editing and drafting the manuscript. C-JW and J-ZW performed all data and econometric analysis and contributed to modelling and drafting the manuscript. G-MZ improved the quality of our manuscript. All authors read and approved the final manuscript.

\footnotetext{
Acknowledgements

This research was supported by the Fundamental Research Funds for the Central Universities (BLYJ201606), the National Natural Science Foundation of China (31270253) and the entrusted project of protection division under State Forestry Bureau "The gap analysis and establishment of regulatory database for three important endangered plant species". We thank the following National Nature Reserves for the use of their species data: Banqiao, Gujingyuan, Qingliangfeng, Songshan, Daiyunshan, E'meifeng, Longqishan, Minjianghekoushidi, Minjiangyuan, Tingjiangyuan, Xiongjianghuangchulin, Zhangjiangkouhongshulinshidi, Gansulianhuashan, Qinzhouzhenxishuishengyeshengdongwu, Taizishan, Yuhe, Haifengniaolei, Lianzhoutianxin, Luokeng'exi, Shimentai, Xiangtoushan, Yunkaishan, Bangliangchangbiyuan, Chongzuobaitouyehou, Daguishan'exi, Dayaoshan, Encheng, Fangchengjinhuacha, Huaping, Jiuwanshan, Qichong, Shiwandashan, Yinzhulaoshanziyuanlengshan, Yuanbaoshan, Dashahe, Fodingshan, Leigongshan, Yinggeling, Changlihuangjinhaian, Hengshuihu, Qingyazhai, Tuoliang, Xiaowutaishan, Baotianman, Henandabieshan, Gaoleshan, Huangheshidi, Jigongshan, Beijicun, Zhuonahe, Daxiagu, Mudanjiangdongbeihu, Dongfanghong, Duobuku'er, Fenglin Heilongjiangfenghuangshan, Gongbielahe, Lingfeng, Maolangou, Mingshui, Mudanfeng, Pingdingshan, Qixingdongbeihu, Sanhuanpao, Shankou, Taipinggou, Wuyiling, Wuyu'erhe, Wudalianchi, Xiaobeihu, Xinqingbaitouhe, Youhao, Zhongyangzhanheizuisongji, Badongjinsihou, Duheyuan, Hubeidabieshan, Mulinzi, Nanhe, Qizimeishan, Saiwudang, Sanxiadalaoling, Shennongjia, Shibalichangxia, Wudaoxia, Xianfengzhongjianhedani, Xingdoushan, Yerengou, Baiyunshan, Dong'anshunhuangshan, Dongdongtinghu, Gaowangjie, Hupingshan, Jintongshan, Jiuyishan, Wuyunjie, Xidongtinghu, Baishanyuanshe, Boluohu, Hunchundongbeihu, Ji'an, Jingyu, Shihu, Wangqing, Yanminghu, Dafengmilu, Yanchengshidizhenqin, Ganjiangyuan, Jiulingshan, Lushan, Qiyunshan, Tongboshan, Wuyuansenlinniaolei, Yangjifeng,
} 
Bailiangshan, Daheishan, Hongluoshan, Louzishan, Nulu'erhushan, Qinglonghe, Shedaolaotieshan, Yalujiangkoushidi, Zhanggutai, A'lu, Bilahe, Gaogesitaihanwula, Hanshan, Hanma, Qingshan, Wulanba, Datongbeichuanheyuanqu, Huanghesanjiaozhou, Nansihu, Heichashan, Lingkongshan, Guanyinshan, Hanchenghuanglongshanhemaji, Huangbaiyuan, Huanglongshanhemaji, Luoyangzhenxishuishengdongwu, Micangshan, Motianling, Pingheliang, Taibaishan, Taibaixushuihe, Wuliangshan, Zhouzhilaoxiancheng, Anzihe, Baihe, Caopo, Gexigou, Heizhugou, Jiudingshan, Laojunshan, Liziping, Nuoshuihezhenxishuishengdongwu, Qianfoshan, Xiaozhaizigou, Xuebaoding, Ailaoshan, Daweishan, Jiaozishan, Lvchunhuanglianshan, Nan'gunhe, Tongbiguan, Wenshan, Wumengshan, Yuanjiang, Yunlongtianchi, Jiushanliedao, Wuyanling, Changxingyangzi'e, Dabashan, Jinfoshan, Wulipo, and Xuebaoshan.

\section{Competing interests}

The authors declare that they have no competing interests.

Received: 15 October 2015 Accepted: 15 June 2016

Published online: 27 June 2016

\section{References}

Adhikari D, Barik SK, Upadhaya K (2012) Habitat distribution modelling for reintroduction of Ilex khasiana Purk., a critically endangered tree species of northeastern India. Ecol Eng 40:37-43

Araújo MB, Alagador D, Cabeza M, Nogués-Bravo D, Thuiller W (2011) Climate change threatens European conservation areas. Ecol Lett 14:484-492

Bradford MA, Warren RJ (2014) Terrestrial biodiversity and climate change. Global Environ Chang 1:355-361

Calabrese JM, Certain G, Kraan C, Dormann CF (2014) Stacking species distribution models and adjusting bias by linking them to macroecological models. Global Ecol Biogeogr 23:99-112

Cao Y, DeWalt RE, Robinson JL, Tweddale T, Hinz L, Pessino M (2013) Using Maxent to model the historic distributions of stonefly species in Illinois streams: the effects of regularization and threshold selections. Ecol Model 259:30-39

Chape S, Harrison J, Spalding M, Lysenko I (2005) Measuring the extent and effectiveness of protected areas as an indicator for meeting global biodiversity targets. Philos Trans R Soc B 360:443-455

Chen YH (2007) Prioritizing avian conservation areas in china by hotspot scoring, heuristics and optimisation. Acta Ornithol 42:119-128

Chen YH (2013) Habitat suitability modeling of amphibian species in southern and central china: environmental correlates and potential richness mapping. Sci China Life Sci 56:476-484

Chung MY, López-Pujol J, Chung JM, Kim KJ, Park SJ, Chung MG (2015) Polyploidy in Lilium lancifolium: evidence of autotriploidy and no niche divergence between diploid and triploid cytotypes in their native ranges. Flora 213:57-68

Dawson TP, Jackson ST, House JI, Prentice IC, Mace GM (2011) Beyond predictions: biodiversity conservation in a changing climate. Science 332:53-58

Di Minin E, Moilanen A (2012) Empirical evidence for reduced protection levels across biodiversity features from targetbased conservation planning. Biol Conserv 153:187-191

Di Minin E, Moilanen A (2014) Improving the surrogacy effectiveness of charismatic megafauna with well-surveyed taxonomic groups and habitat types. J Appl Ecol 51:281-288

Di Minin E, Macmillan DC, Goodman PS, Escott B, Slotow R, Moilanen A (2013) Conservation businesses and conservation planning in a biological diversity hotspot. Conserv Biol 27:808-820

Elith J, Phillips SJ, Hastie T, Dudík M, Chee YE, Yates CJ (2011) A statistical explanation of MaxEnt for ecologists. Divers Distrib 17:43-57

Gillson L, Dawson TP, Jack S, McGeoch MA (2013) Accommodating climate change contingencies in conservation strategy. Trends Ecol Evol 28:135-142

Graf M (1999) Plants of the Tahoe basin: flowering plants, trees, and ferns - a photographic guide. University of California Press, California

Hampe A, Petit RJ (2005) Conserving biodiversity under climate change: the rear edge matters. Ecol Lett 8:461-467

Hannah L, Midgley GF, Millar D (2002) Climate change-integrated conservation strategies. Global Ecol Biogeogr 11:485-495

Heller NE, Zavaleta ES (2009) Biodiversity management in the face of climate change: a review of 22 years of recommendations. Biol Conserv 142:14-32

Hou X (2001) Vegetation atlas of China. Chinese Academy of Science. The Editorial Board of Vegetation Map of China. Scientific Press, Beijing

Jackson ST, Sax DF (2010) Balancing biodiversity in a changing environment: extinction debt, immigration credit and species turnover. Trends Ecol Evol 25:153-160

Lawler JJ (2009) Climate change adaptation strategies for resource management and conservation planning. Ann N Y Acad Sci 1162:79-98

Leader-Williams N, Albon SD (1988) Allocation of resources for conservation. Nature 336:533-535

Lehtomäki J, Moilanen A (2013) Methods and workflow for spatial conservation prioritization using Zonation. Environ Model Softw 47:128-137

Li W, Guo Q (2013) How to assess the prediction accuracy of species presence-absence models without absence data? Ecography 36:788-799

Meller L, Cabeza M, Pironon S, Barbet-Massin M, Maiorano L, Georges D, Thuiller W (2014) Ensemble distribution models in conservation prioritization: from consensus predictions to consensus reserve networks. Divers Distrib 20:309-321

Merow C, Smith MJ, Silander JA (2013) A practical guide to MaxEnt for modeling species' distributions: what it does, and why inputs and settings matter. Ecography 36:1058-1069 
Pearson RG, Dawson TP (2003) Predicting the impacts of climate change on the distribution of species: are bioclimate envelope models useful? Global Ecol Biogeogr 12:361-371

Pearson RG, Raxworthy CJ, Nakamura M, Townsend Peterson A (2007) Predicting species distributions from small numbers of occurrence records: a test case using cryptic geckos in Madagascar. J Biogeogr 34:102-117

Pouteau R, Bayle É, Blanchard É, Birnbaum P, Cassan J, Hequet V, Ibanez T, Vandrot H (2015) Accounting for the indirect area effect in stacked species distribution models to map species richness in a montane biodiversity hotspot. Divers Distrib 21:1329-1338

Ravenel RM, Redford KH (2005) Understanding IUCN protected area categories. Nat Area J 25:381-389

Royle JA, Chandler RB, Yackulic C, Nichols JD (2012) Likelihood analysis of species occurrence probability from presenceonly data for modelling species distributions. Methods Ecol Evol 3:545-554

Sgro CM, Lowe AJ, Hoffmann AA (2011) Building evolutionary resilience for conserving biodiversity under climate change. Evol Appl 4:326-337

Sharafi SM, Moilanen A, White M, Burgman M (2012) Integrating environmental gap analysis with spatial conservation prioritization: a case study from Victoria, Australia. J Environ Manag 112:240-251

Shcheglovitova M, Anderson RP (2013) Estimating optimal complexity for ecological niche models: a jackknife approach for species with small sample sizes. Ecol Model 269:9-17

Summers DM, Bryan BA, Crossman ND, Meyer WS (2012) Species vulnerability to climate change: impacts on spatial conservation priorities and species representation. Global Change Biol 18:2335-2348

Wan J, Wang C, Han S, Yu J (2014) Planning the priority protected areas of endangered orchid species in northeastern China. Biodivers Conserv 23:1395-1409

Wan J, Wang C, Yu J, Nie S, Han S, Wang L, Liu J, Zu Y (2015) Model-based assessment of priority protected areas: a case study on Fraxinus mandshurica in China. Pol J Environ Stud 24:725-733

Wan J, Wang C, Yu J, Nie S, Han S, Liu J, Zu Y, Wang Q (2016) Developing conservation strategies for Pinus koraiensis and Eleutherococcus senticosus by using model-based geographic distributions. J For Res 27:389-400

Wang CJ, Wan JZ, Mu XY, Zhang ZX (2015) Management planning for endangered plant species in priority protected areas. Biodivers Conserv 24:2383-2397

Wang CJ, Wan JZ, Zhang GM, Zhang ZX, Zhang J (2016) Protected areas may not effectively support conservation of endangered forest plants under climate change. Environ Earth Sci 75:1-9

Warren DL, Seifert SN (2011) Ecological niche modeling in Maxent: the importance of model complexity and the performance of model selection criteria. Ecol Appl 21:335-342

Yu J, Wang C, Wan J, Han S, Wang Q, Nie S (2014) A model-based method to evaluate the ability of nature reserves to protect endangered tree species in the context of climate change. For Ecol Manag 327:48-54

Zhang L, Xu WH, Ouyang ZY, Zhu CQ (2014a) Determination of priority nature conservation areas and human disturbances in the Yangtze River Basin, China. J Nat Conserv 22:326-336

Zhang MG, Zhou ZK, Chen WY, Cannon CH, Raes N, Slik JW (2014b) Major declines of woody plant species ranges under climate change in Yunnan, China. Divers Distrib 20:405-415

\section{Submit your manuscript to a SpringerOpen ${ }^{\circ}$ journal and benefit from:}

- Convenient online submission

- Rigorous peer review

- Immediate publication on acceptance

Open access: articles freely available online

High visibility within the field

Retaining the copyright to your article

Submit your next manuscript at $\boldsymbol{\nabla}$ springeropen.com 